\title{
Prevalence and natural history of adrenal incidentalomas - a prospective cohort study
}

\author{
Panagiotis Anagnostis, Athanasios Siolos, Fotini Adamidou, Chrisanthi Zouli, \\ Gesthimani Mintziori, Zoe Efstathiadou, Athanasios Panagiotou, Marina Kita
}

\section{Department of Endocrinology and Diabetes, Hippokration General Hospital of Thessaloniki, Greece}

\section{Introduction}

\begin{abstract}
$>$ Adrenal incidentalomas (Als) are frequently detected due to the increasing use of abdominal imaging for diagnostic purposes.
\end{abstract}

Few prospective studies exist with respect to their natural history, regarding their size and clinical course.

\section{Aims}

The aim of this study was to report the prevalence and natural history of Als.

\section{Patients and methods}

Interim-analysis of a prospective cohort study, conducted at the department of Endocrinology in Hippokration General Hospital (Thessaloniki, Greece), a tertiary referral centre for endocrinology and diabetes.

\section{Hormonal assessment:}

$\checkmark$ Serum cortisol nocturnal rhythm, 24h-urinary free cortisol, low-dose dexamethasone-suppression-test

Aldosterone to plasma-renin-activity ratio

$\checkmark 24$ h-urinary total metanephrines and catecholamines

In cases of positive results, confirmatory tests were performed.

\section{Radiological assessment (CT or MRI)}

At 6-12 months and yearly thereafter

\section{Results}

64 patients [19 (30\%) males, mean age $59.7 \pm 12.1$ years) were included (median follow-up time: 36 months, range 484).

Median size: $2.5 \mathrm{~mm}$ (range 0.5-6.5) [unilateral adenoma in $50(78 \%)$.

Final diagnosis was:

- 56 non-functioning adenomas (87.5\%),

- $5(7.8 \%)$ subclinical Cushing's syndrome (SCS)

-1 1 (1.6\%) pheochromocytoma

$-1(1.6 \%)$ aldosteronoma

-2 (3.2\%), adrenocortical carcinomas, one of which had also SCS.

\section{Natural history}

Mass enlargement $(9 \mathrm{~mm})$ was observed in one patient $(2.8 \%)$, while a decrease $(7-27 \mathrm{~mm})$ in three $(8.5 \%)$.

No hormonal evolution was noticed.

\section{Clinical outcomes}

Regarding glucose metabolism, $12 \%, 16.7 \%$ and $20 \%$ of the patients presented deterioration in glucose

metabolism at the first, second and third year of followup, respectively.

The respective percentages for dyslipidemia were $26 \%$, $43 \%$ and $44 \%$ and for hypertension or blood pressure worsening were $38 \%, 44 \%$ and $48 \%$.

After excluding those with SCS, these percentages did not alter significantly.

New cardiovascular events or fractures were recorded in $7.8 \%$ of the patients.

\section{Conclusions}

\section{References}

Anagnostis P, et al. Hormones 2009;8:163-184.

Anagnostis $\mathrm{P}$, et al. Exp Clin Endocrinol Diabetes. 2010;118:610-6.

Terzolo M, et al. Eur J Endocrinol. 2011;164:851-70.

Zeiger MA, et al. Endocr Pract. 2009;15 Suppl 1:1-20.
The vast majority of Als involved benign, non-secretory masses.

Mass enlargement was rare.

$>1 / 3$ of patients developed hypertension or their lipid profile worsened, while $1 / 5$ showed deterioration in glucose metabolism, irrespectively of SCS. 